\title{
High-pressure experiments and modeling of methane/air catalytic combustion for power-generation applications
}

\author{
Richard Carroni $^{\mathrm{a}, *}$, Timothy Griffin ${ }^{\mathrm{a}}$, John Mantzaras ${ }^{\mathrm{b}}$, Michael Reinke ${ }^{\mathrm{b}}$ \\ a ALSTOM Power, Technology Center, CH-5405 Baden-Dättwil, Switzerland \\ ${ }^{\mathrm{b}}$ Paul Scherrer Institute, Combustion Research, CH-5232 Villigen PSI, Switzerland
}

Received 1 May 2002; accepted 10 December 2002

\begin{abstract}
The catalytic combustion of methane/air mixtures is investigated experimentally and numerically at gas turbine relevant conditions (inlet temperatures up to $873 \mathrm{~K}$, pressures up to $15 \mathrm{bar}$ and spatial velocities up to $3 \times 10^{6} \mathrm{~h}^{-1}$ ). Experiments are performed in a sub-scale test rig, consisting of a metallic honeycomb structure with alternately coated (Pd-based catalyst) channels. Simulations are carried out with a two-dimensional elliptic fluid mechanical code that incorporates detailed transport and heat loss mechanisms, and realistic heterogeneous and homogeneous chemistry description. The methodology for extracting heterogeneous kinetic data from the experiments is presented, and the effects of catalytic activity and channel geometry (length and hydraulic diameter) on reactor performance are elucidated. A global catalytic kinetic step provides excellent agreement (at temperatures below $950 \mathrm{~K}$ ) between the measured and predicted fuel conversion, over a wide range of parameter variation (channel hydraulic diameter and length, pressure, and inlet temperature). It is shown that, under a certain combination of catalytic activity and channel length, the absolute temperature rise across the catalyst becomes essentially independent of pressure, a feature highly desirable for many practical systems. Even though the computed catalyst surface temperatures remain well below the decomposition temperature of $\mathrm{PdO}$, a significant section of the catalyst-amounting up to $30 \%$ of the total reactor length-contributes minimally to the total fuel conversion, suggesting catalytic activity design improvements in the reactor entry.
\end{abstract}

(c) 2003 Elsevier B.V. All rights reserved.

Keywords: Catalytically stabilized combustion; Gas turbines

\section{Introduction}

Considerable efforts have been devoted to the research of catalytic combustors for gas turbine applications, in an effort to further reduce thermal $\mathrm{NO}_{x}$ emissions. Given the complexity and cost of developing such systems, it is desirable to complement experimental work with numerical simulations. Modeling tools aid with the planning and interpretation of

\footnotetext{
* Corresponding author.

E-mail address: richard.carroni@power.alstom.com (R. Carroni).
}

experiments, and greatly facilitate the overall design of catalytic combustors. The tools are necessarily complex, for they must accurately describe a number of interacting phenomena in fluid mechanics, heat transfer and chemistry (both heterogeneous and homogeneous). Occasionally, the physics lends itself to simpler descriptions. In many cases, for example, atmospheric tests of Pd-based catalysts can be simulated reasonably well by assuming mass-transfer controlled surface reactions. At high pressures, though, this assumption often yields fuel conversions that are several times larger than measured values, thereby implying 


\begin{tabular}{|c|c|}
\hline \multicolumn{2}{|c|}{ Nomenclature } \\
\hline$c_{p}$ & specific heat at constant pressure \\
\hline$D_{\mathrm{h}}$ & channel hydraulic diameter (Fig. 2) \\
\hline$D_{k}$ & $\begin{array}{l}\text { mixture-average species diffusion } \\
\text { coefficient (Eq. (6)) }\end{array}$ \\
\hline$E$ & activation energy \\
\hline$f$ & skin friction factor \\
\hline$h$ & total enthalpy (Eq. (4)) \\
\hline$h_{k}^{0}$ & $\begin{array}{l}\text { chemical enthalpy of } k \text { th species } \\
\text { (Eq. (7)) }\end{array}$ \\
\hline$k$ & reaction rate coefficient (Eq. (11)) \\
\hline$K_{\mathrm{g}}$ & total number of gas-phase species \\
\hline$L^{0}$ & channel length (Fig. 2) \\
\hline$N u_{\mathrm{H}}$ & $\begin{array}{l}\text { Nusselt number, constant heat } \\
\text { transfer case }\end{array}$ \\
\hline$p$ & pressure \\
\hline$\dot{q}_{\mathrm{rad}}$ & radiative heat transfer (Eq. (8)) \\
\hline$r$ & radial co-ordinate \\
\hline$R$ & universal gas constant \\
\hline$R_{\mathrm{C}}$ & surface reaction rate (Eq. (11)) \\
\hline$R_{\mathrm{h}}$ & channel hydraulic radius (Fig. 2) \\
\hline$R e$ & Reynolds number \\
\hline$\dot{s}_{k}$ & $\begin{array}{l}\text { species heterogeneous molar production } \\
\text { rates (Eqs. (8) and (9)) }\end{array}$ \\
\hline$T$ & temperature \\
\hline$T_{0}$ & reference temperature (Eq. (7)) \\
\hline$u, U_{\text {in }}$ & $\begin{array}{l}\text { streamwise velocity and inlet } \\
\text { streamwise velocity, respectively }\end{array}$ \\
\hline$v$ & transverse velocity \\
\hline$\vec{V}_{k}$ & $\begin{array}{l}\text { species diffusion velocity vector } \\
\text { (Eq. (6)) }\end{array}$ \\
\hline$W_{k}$ & gas-phase species molecular weight \\
\hline $\bar{W}$ & $\begin{array}{l}\text { average gaseous mixture molecular } \\
\text { weight }\end{array}$ \\
\hline$x$ & streamwise co-ordinate \\
\hline$Y_{k}$ & gas-phase species mass fraction \\
\hline \multicolumn{2}{|c|}{ Greek symbols } \\
\hline$\delta$ & channel wall thickness (Fig. 2) \\
\hline$\Delta T$ & $\begin{array}{l}\text { temperature rise of gas at channel } \\
\text { exit }\left(=T_{L, \mathrm{~m}}-T_{\text {in }}\right)\end{array}$ \\
\hline$\eta$ & conversion $\left(=100 \times \Delta T /\left(T_{\mathrm{ad}}-T_{\mathrm{in}}\right)\right)$ \\
\hline$\theta_{T, k}$ & species thermal diffusion ratio (Eq. (6)) \\
\hline$\lambda_{\mathrm{g}}$ & thermal conductivity of the gas (Eq. (8)) \\
\hline$\lambda_{\mathrm{s}}^{\mathrm{s}}$ & thermal conductivity of the solid (Eq. (8)) \\
\hline$v$ & kinematic viscosity \\
\hline$\rho$ & density \\
\hline
\end{tabular}

\begin{tabular}{|ll|}
\hline$\sigma$ & Stefan-Boltzmann constant (Eq. (10)) \\
$\varphi$ & equivalence ratio \\
$\dot{\omega}_{k}$ & gas-phase species molar production \\
& rate (Eq. (5)) \\
\multicolumn{2}{|l|}{ Subscripts } \\
in & inlet \\
$\mathrm{L}$ & exit \\
$\mathrm{W}$ & wall \\
$x, r$ & streamwise and radial components \\
& (Eq. (4)) \\
\hline
\end{tabular}

that heterogeneous processes at gas turbine conditions are largely influenced by kinetics. The effectiveness of the modeling tools thus depends strongly on the availability of reliable, heterogeneous, kinetic data, which are necessary for the correct description of the catalytic processes. In gas turbine applications, the catalysts of choice are palladium-based (at least for low and intermediate temperatures) because $\mathrm{Pd}$ is the most active catalyst for natural gas. It is a well-known fact that palladium undergoes complex temperatureand pressure-dependent transformations, which significantly affect its ability to convert fuel over a wide range of operating conditions. A number of multi-step reaction schemes have recently attempted to describe the oxidation of commercially interesting fuels on palladium: Deutschmann et al. [1] for hydrogen, Moallemi et al. [2] and Zhu and Jackson [3] for methane. It is understood, however, that the emphasis of these schemes was on low-temperature, low-pressure catalytic performance, without aiming to resolve the complicated $\mathrm{PdO}$ decomposition and $\mathrm{Pd}$ re-oxidation processes observed in experiments [4].

Given the importance of heterogeneous kinetics in the underlying processes, a concerted effort was made to extract the necessary information from highpressure, steady-state, catalytic tests performed under conditions relevant to gas turbines. Numerical simulations were carried out using a two-dimensional, elliptic CFD code capable of treating detailed, hetero/homogeneous chemistry, fluid transport and various heat transfer mechanisms. The aim was to deduce global, heterogeneous, kinetic data that can reproduce a wide range of experiments and subsequently use the validated model for detailed parametric studies and, eventually, for design purposes. Of particular interest was 
the assessment of the advantages/disadvantages of specific catalysts as potential candidates for different sectors of the catalytically stabilized combustion (CST) burner, and their implications on the characteristics of the reactor geometry. More specifically, it was desirable to quantify the fraction of catalytic reactor length encompassed by the steady-state light-off distance.

\section{Experimental work}

\subsection{Reactor configuration}

The high-pressure test rig (rated at $>30$ bar) used at ALSTOM Power was of a modular design, thereby providing the platform for investigating various combustion systems. It consisted of an electric heating unit, a mixing section, a test module, a burnout zone and a cooling/exhaust pipe (see Fig. 1a). Three $30 \mathrm{~kW}$ electric heaters preheated air (or other oxidant carriers, such as air + steam or air +EG) to the desired temperature. A $30 \mathrm{~cm}$ long series of static mixers allowed for high levels of fuel/air premixedness, which is a prerequisite for stable catalytic operation free of hot-spots. The test section and burnout zones were lined with ceramic insulation in order to promote near-adiabatic operation. System pressure and velocity was regulated via a throttle, which ensured that the flow was always choked, thereby simplifying the relationship between velocity, pressure and mass flow.

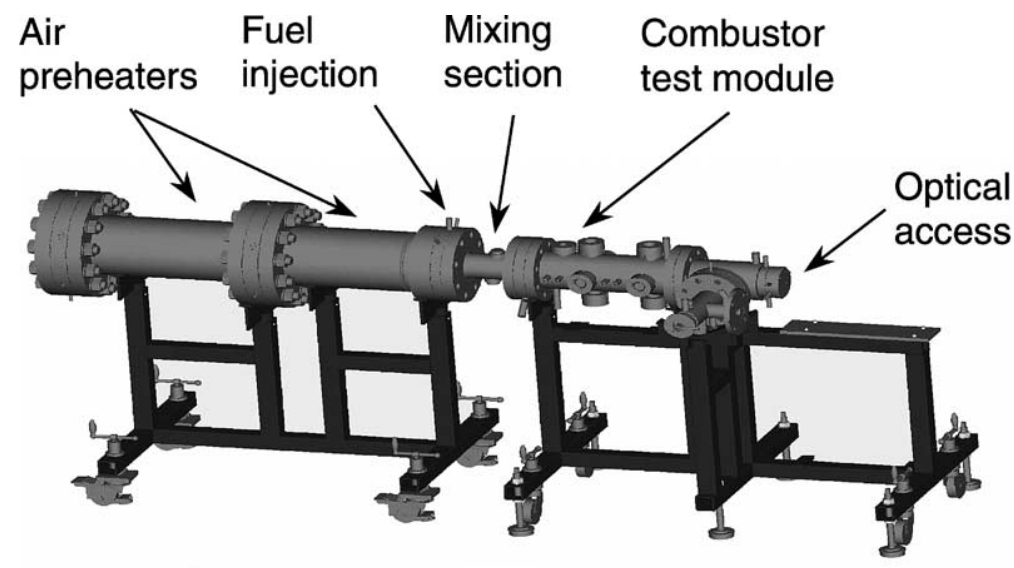

(a)

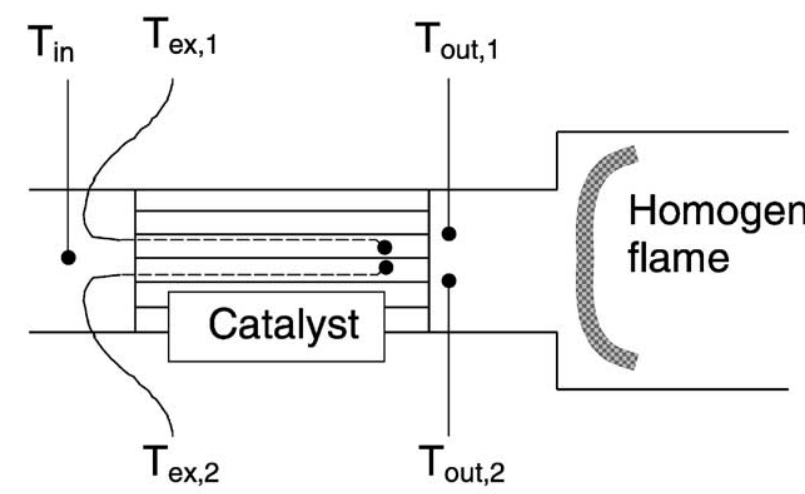

(b)

Fig. 1. (a) High-pressure test rig configuration, and (b) schematic illustration of the catalytic test module, indicating the temperature measurement locations. Test rig operating parameters: maximum flow rate: $250 \mathrm{~g} / \mathrm{s}$, pressure: $1-35 \mathrm{bar}$, maximum thermal power: $300 \mathrm{~kW}$, air preheat temperature: $0-650^{\circ} \mathrm{C}$. 
A homogeneous flame converted the remaining fuel in a post-catalyst combustion zone, positioned downstream of the catalyst, in order to stabilize the system in terms of pressure and flow rate fluctuations, which can otherwise lead to catalyst destruction. Anchoring of the homogeneous flame was attained via the recirculation zone created by a sudden expansion (area expansion ratio $=3.8$ ). A $100 \mathrm{~mm}$ gap separated the catalyst exit plane from the expansion, in order to minimize the impact of the flame upon the catalyst.

Fig. 1b depicts the catalytic test module and indicates the locations at which temperatures were measured. $T_{\mathrm{ex}, 1}$ and $T_{\mathrm{ex}, 2}$ are local catalyst exit temperatures measured with K-type thermocouples, inserted via the inlet face and extended all the way (through uncoated channels, whenever possible) to the exit plane. $T_{\text {out, } 1}$ and $T_{\text {out,2 }}$ (located approximately $10 \mathrm{~mm}$ downstream of the catalyst) provided a more reliable measurement of overall catalyst exit temperature; these values were used to compute the temperature rise over the catalyst $\left(\Delta T_{1}=T_{\text {out } 1}-T_{\text {in }}\right.$ and $\left.\Delta T_{2}=T_{\text {out }, 2}-T_{\text {in }}\right)$. The inlet temperature $T_{\text {in }}$ was measured up to $100 \mathrm{~mm}$ (depending on catalyst length) upstream of the catalyst inlet; despite this, adiabaticity was confirmed by the inlet and exit temperatures being equal $\left(T_{\text {in }}=T_{\text {out }, 1}=T_{\text {out }, 2}\right)$ when only hot air (>673 K) was passed through the system. Since only heterogeneous combustion occurred within the catalytic section without significant gas-phase contribution (see discussion on numerical modeling), the catalytic conversion was derived by comparing the average temperature rise $\Delta T=(1 / 2)\left(\Delta T_{1}+\Delta T_{2}\right)$ with the potential adiabatic temperature gain: $\eta=$ $\Delta T /\left(T_{\mathrm{ad}}-T_{\mathrm{in}}\right)$. In all examined cases the length of the catalytic reactor was not sufficient to achieve complete fuel conversion inside each coated channel, even under the assumption of mass-transport-limited operation; therefore, the attained $\Delta T$ is preferably compared against the maximum possible attainable mass-transport-limited conversion $\Delta T_{\max }$. This ratio is further denoted as the fractional fuel conversion.

The catalytic reactor itself comprised a multitude of alternately coated channels which formed a cylindrical structure $35 \mathrm{~mm}$ in diameter, with a length $(L)$ of either 64 or $136 \mathrm{~mm}$. The structure (see Fig. 2a) consisted of a succession of corrugated and flat $\mathrm{FeCr}$-alloy foils of thickness $\delta=51 \mu \mathrm{m}$, each coated only on one side, thus creating a sequence of trapezoidal, catalyti-

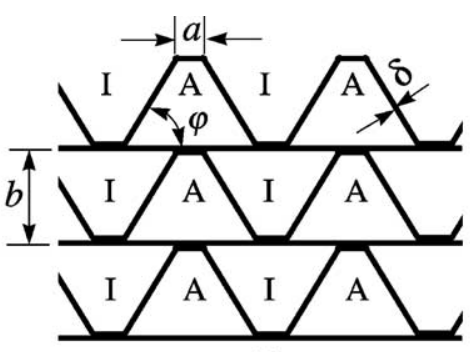

(a)

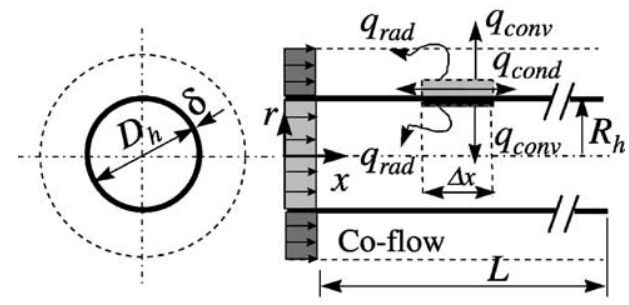

(b)

Fig. 2. (a) Schematic illustration of the alternately catalytically active (A) and inactive (I) trapezoidal channels, which are formed with a sequence of corrugated and flat FeCr-alloy foils coated only on one side, and (b) the equivalent cylindrical channel used in the simulations and the modeled heat transfer processes in each individual differential cylindrical element of length $\Delta x$.

cally active and catalytically inert channels. The flow in the inert channels provided cooling for the active channels such that the catalyst surface temperature was maintained at acceptable levels. Two different channel structures were used, with equivalent hydraulic diameters $\left(D_{\mathrm{h}}\right)$ of 1 and $1.2 \mathrm{~mm}$; both were characterized by the same geometric ratio $a / b$ and angle $\varphi$ (see Fig. 2a).

\subsection{Test conditions and procedure}

Two different PdO-based catalysts on alumina washcoats, denoted as A and B, were applied to the $\mathrm{FeCr}$ alloy. Channel hydraulic diameters of 1 and $1.2 \mathrm{~mm}$ were utilized, respectively. The form of the catalyst kinetic expression was determined by the supplier (see details in Section 3.3), with the aid of an isothermal characterization of the catalyst powder in a fixed-bed reactor. Catalyst A was tested only on a $64 \mathrm{~mm}$ long structure, whilst sample B was investigated on both 64 and $136 \mathrm{~mm}$ long supports.

The catalytic reactors were subjected to conditions similar to those found in modern gas turbine combustors, using $\mathrm{CH}_{4}$ as the fuel. Experiments were 
Table 1

Experimental conditions ${ }^{\mathrm{a}}$

\begin{tabular}{lllrllll}
\hline Case & Catalyst & $\begin{array}{l}L \\
(\mathrm{~mm})\end{array}$ & \multicolumn{1}{c}{$\begin{array}{l}p \\
(\mathrm{bar})\end{array}$} & $\varphi$ & $\begin{array}{l}U_{\text {in }} \\
(\mathrm{m} / \mathrm{s})\end{array}$ & $\begin{array}{l}T_{\text {in }} \\
(\mathrm{K})\end{array}$ & $R e_{\text {in }}$ \\
\hline $\mathrm{A} 1$ & $\mathrm{~A}$ & 64 & 5 & 0.40 & 15 & 723 & 1144 \\
$\mathrm{~A} 2$ & $\mathrm{~A}$ & 64 & 10 & 0.40 & 15 & 723 & 2288 \\
$\mathrm{~B} 1$ & $\mathrm{~B}$ & 64 & 5 & 0.40 & 15 & 698 & 1335 \\
$\mathrm{~B} 2$ & $\mathrm{~B}$ & 136 & 5 & 0.40 & 15 & 698 & 1335 \\
$\mathrm{~B} 3$ & $\mathrm{~B}$ & 136 & 5 & 0.40 & 15 & 723 & 1258 \\
$\mathrm{~B} 4$ & $\mathrm{~B}$ & 136 & 10 & 0.40 & 15 & 723 & 2516 \\
$\mathrm{~B} 5$ & $\mathrm{~B}$ & 136 & 15 & 0.40 & 15 & 723 & 3774
\end{tabular}

${ }^{\text {a }}$ Pressure $(p)$, fuel-to-air equivalence ratio $(\varphi)$, inlet velocity $\left(U_{\text {in }}\right)$, inlet temperature $\left(T_{\text {in }}\right)$ and inlet Reynolds number $\left(R e_{\text {in }}=\right.$ $\left.U_{\text {in }} D_{\mathrm{h}} / n_{\text {in }}\right)$. The hydraulic diameter was $D_{\mathrm{h}}=1 \mathrm{~mm}$ in cases A1 and A2 (catalyst A) and $D_{\mathrm{h}}=1.2 \mathrm{~mm}$ in B1-B5 (catalyst B). The inlet velocities are referenced to $723 \mathrm{~K}$ inlet temperature.

performed using an inlet velocity of $15 \mathrm{~m} / \mathrm{s}$ (at about $723 \mathrm{~K}$ ), pressures of 5, 10 and $15 \mathrm{bar}$ and a fuel/air equivalence ratio $\phi=0.40$. Testing essentially consisted of monitoring catalyst performance (conversion and stability characteristics) over longer (i.e. minutes) periods of time, for incremental changes in inlet temperature, at a given pressure and velocity. A number of runs were performed at a given set of conditions, in order to check for repeatability of the results. The conditions used in the present study are summarized in Table 1.

All flow conditions within the catalytic reactor channels were laminar (as determined by the inlet Reynolds numbers), with the exception of the transitional case B5. Nevertheless, recent studies on turbulent, CST combustion [5,6] have shown that under the strong flow laminarization induced by heat transfer from the hot catalyst surfaces, laminar modeling remains a very good approximation, even with incoming Reynolds numbers up to 6000 .

\section{Numerical work}

The platform upon which the numerical activities were performed was a two-dimensional, elliptic CFD code capable of treating detailed hetero/homogeneous chemistry, transport, and various modes of heat transfer. Details of the numerical code are provided elsewhere [5,7]. Each catalytic, trapezoidal channel was modeled as an equivalent cylindrical channel with the same hydraulic diameter and wall thickness (see Fig. 2b). This was a reasonable simplification for the channel geometry of Fig. 2a; the values $a / b=0.2$ and $\varphi \approx 68$ meant that the friction factors $f \times R e$ and Nusselt numbers $N u_{\mathrm{H}}$ were within 6 and $15 \%$ of the corresponding values of a cylindrical duct (see [8]). These discrepancies lie well within the experimental and numerical uncertainties of the present investigation. Moreover, the 2D axisymmetric modeling rendered the computations tractable. The steady-state processes modeled in each differential, cylindrical, surface element of length $\Delta x$ are shown in Fig. 2b, and included: axial heat conduction in the solid FeCr-alloy $\left(\lambda_{\mathrm{s}}=16 \mathrm{~W} \mathrm{~m}^{-1} \mathrm{~K}^{-1}\right)$, two-sided radiation heat exchange, heat generation via surface reactions and, finally, two-sided convection. The local convective heat losses from the outer channel surface were considered to be equal to those from the inner, active side. This required nearly equal local surface temperatures on both sides of the foil, and equal, local, convective flows and heat-transfer film resistances on both foil sides. These approximations were indeed valid under steady-state operating conditions. The radial heat resistance was small (given the small wall thickness $\delta$ and large thermal conductivity $\lambda_{\mathrm{s}}$ of the $\mathrm{FeCr}$ alloy) and hence there were no significant radial temperature gradients in the solid. Furthermore, the $\mathrm{CH}_{4} /$ air flow surrounding the channel had, at any given axial location $x$, approximately the same mean axial velocity as the inner flow at that position. This similarity in velocity was a result of the active and inactive channels having the same geometry, and of the very small change in $\lambda_{\mathrm{g}}$ and $c_{p}$ during lean $(\varphi=0.40) \mathrm{CH}_{4} /$ air combustion. For a typical inlet temperature of $723 \mathrm{~K}$, the reactants and products were characterized by $\lambda_{\mathrm{g}}=0.0553 \mathrm{~W} \mathrm{~m}^{-1} \mathrm{~K}^{-1}$ and $c_{p}=$ $1.144 \mathrm{~kJ} \mathrm{~kg}^{-1} \mathrm{~K}^{-1}$, and $\lambda_{\mathrm{g}}=0.0548 \mathrm{~W} \mathrm{~m}^{-1} \mathrm{~K}^{-1}$ and $c_{p}=1.145 \mathrm{~kJ} \mathrm{~kg}^{-1} \mathrm{~K}^{-1}$, respectively. At the corresponding adiabatic flame temperature $(1625 \mathrm{~K}), \lambda_{\mathrm{g}}=$ $0.1079 \mathrm{~W} \mathrm{~m}^{-1} \mathrm{~K}^{-1}$ and $c_{p}=1.329 \mathrm{~kJ} \mathrm{~kg}^{-1} \mathrm{~K}^{-1}$ for the reactants and $\lambda_{\mathrm{g}}=0.1083 \mathrm{~W} \mathrm{~m}^{-1} \mathrm{~K}^{-1}$ and $c_{p}=1.319 \mathrm{~kJ} \mathrm{~kg}^{-1} \mathrm{~K}^{-1}$ for the products. The local radiation in each differential, cylindrical element of length $\Delta x$ included heat exchange with the inlet, the outlet, and the other differential, cylindrical elements; the local radiation from the outer surface was taken as being equal to that of the inner surface, given the same wall temperature and channel geometry. 


\subsection{Governing equations and boundary conditions}

The governing equations in their steady, elliptic form, written in cylindrical coordinates, were as follows:

Continuity:

$\frac{\partial(\rho u)}{\partial x}+\frac{1}{r} \frac{\partial(r \rho v)}{\partial r}=0$

Momentum (axial, radial):

$$
\begin{aligned}
& \frac{\partial(\text { (ии })}{\partial x}+\frac{1}{r} \frac{\partial(\text { r } \rho v u)}{\partial r} \\
& =-\frac{\partial p}{\partial x}+\frac{\partial}{\partial x}\left[2 \mu \frac{\partial u}{\partial x}-\frac{2}{3} \mu\left(\frac{\partial u}{\partial x}+\frac{1}{r} \frac{\partial(r v)}{\partial r}\right)\right] \\
& \quad+\frac{1}{r} \frac{\partial}{\partial r}\left[\mu r\left(\frac{\partial u}{\partial r}+\frac{\partial v}{\partial x}\right)\right]=0 \\
& \frac{\partial(\rho u v)}{\partial x}+\frac{1}{r} \frac{\partial(r \rho v v)}{\partial r} \\
& =-\frac{\partial p}{\partial r}+\frac{\partial}{\partial x}\left[\mu\left(\frac{\partial v}{\partial x}+\frac{\partial u}{\partial r}\right)\right] \\
& \quad+\frac{\partial}{\partial r}\left[2 \mu \frac{\partial v}{\partial r}-\frac{2}{3} \mu\left(\frac{\partial u}{\partial x}+\frac{1}{r} \frac{\partial(r v)}{\partial r}\right)\right] \\
& \quad+\frac{2 \mu}{r}\left(\frac{\partial v}{\partial r}+\frac{v}{r}\right)-\rho g=0
\end{aligned}
$$

Energy:

$$
\begin{aligned}
& \frac{\partial(\rho u h)}{\partial x}+\frac{\partial(r \rho v h)}{\partial r} \\
& =\frac{\partial}{\partial x}\left(\lambda_{\mathrm{g}} \frac{\partial T}{\partial x}-\rho \sum_{k=1}^{K_{\mathrm{g}}} Y_{k} h_{k} V_{k, x}\right) \\
& \quad+\frac{1}{r} \frac{\partial}{\partial r}\left(r \lambda_{\mathrm{g}} \frac{\partial T}{\partial r}-r \rho \sum_{k=1}^{K_{\mathrm{g}}} Y_{k} h_{k} V_{k, r}\right)=0
\end{aligned}
$$

Species:

$$
\begin{aligned}
& \frac{\partial\left(\rho u Y_{k}\right)}{\partial x}+\frac{1}{r} \frac{\partial\left(r \rho v Y_{k}\right)}{\partial r} \\
& =-\frac{\partial}{\partial x}\left(\rho Y_{k} V_{k, x}\right)-\frac{1}{r} \frac{\partial}{\partial r}\left(r \rho Y_{k} V_{k, r}\right)+\dot{\omega}_{k} W_{k}=0, \\
& \quad k=1,2, \ldots, K_{\mathrm{g}}
\end{aligned}
$$

where

$$
\vec{V}_{k}=-D_{k} \vec{\nabla}\left[\ln \left(\frac{Y_{k} \bar{W}}{W_{k}}\right)\right]+\left[\frac{D_{k} \theta_{T, k} W_{k}}{Y_{k} \bar{W}}\right] \bar{\nabla}(\ln T)
$$

The transport model of Eq. (6) included the mixture-average diffusion as well as the thermal diffusion for the light species [9]. Finally, the ideal gas and caloric equations of state were, respectively:

$p=\frac{\rho R T}{\bar{W}}, \quad h_{k}=h_{k}^{0}\left(T_{0}\right)+\int_{T_{0}}^{T} c_{p, k} \mathrm{~d} T$

\subsection{Boundary conditions}

The interfacial energy boundary condition $\left(r=R_{\mathrm{h}}\right)$ was

$$
\begin{gathered}
2 \dot{q}_{\mathrm{rad}}+\lambda_{\mathrm{s}} \frac{\partial^{2} T}{\partial x^{2}} \delta-2\left(\lambda_{\mathrm{g}} \frac{\partial T}{\partial r}\right)_{r=R_{\mathrm{h}}} \\
+\sum_{k=1}^{K_{\mathrm{g}}}\left(\rho \dot{s}_{k} h_{k} W_{k}\right)_{r=R_{\mathrm{h}}}=0
\end{gathered}
$$

The multiplicative factor of 2 in the radiative and convective heat loss terms (i.e. those containing $\dot{q}_{\text {rad }}$ and $\lambda_{\mathrm{g}}(\partial T / \partial r)$, respectively) represented the influence of the surrounding, inert channels, as discussed within the context of Fig. 2b. The interfacial boundary conditions for the gas-phase species were

$\left(\rho Y_{k} V_{k, r}\right)_{r=R_{\mathrm{h}}}+\dot{s}_{k} W_{k}=0, \quad k=1,2, \ldots, K_{\mathrm{g}}$

Radiative boundary conditions were applied to the vertical faces of the channel:

$$
\begin{aligned}
& \left.\lambda_{\mathrm{s}} \frac{\partial T_{\mathrm{W}}}{\partial x}\right|_{x=0}=\varepsilon \sigma\left(T_{\mathrm{W}, 0}^{4}-T_{\mathrm{in}}^{4}\right) \quad \text { at } x=0, \\
& -\left.\lambda_{\mathrm{s}} \frac{\partial T_{\mathrm{W}}}{\partial x}\right|_{x=L}=\varepsilon \sigma\left(T_{\mathrm{W}, L}^{4}-T_{\mathrm{L}, \mathrm{m}}^{4}\right) \quad \text { at } x=L
\end{aligned}
$$

where $T_{\mathrm{L}, \mathrm{m}}$ is the average gas temperature at the exit. A surface emissivity of $\varepsilon=0.5$ was applied. Uniform profiles for the axial velocity and the scalars were specified at the inlet. Zero-Neumann boundary conditions were applied to the scalars and axial velocity $(\partial \Phi / \partial n=\partial u / \partial n=0$, where $n$ is the direction normal to the corresponding surface) at the axis of symmetry $(r=0)$ and the channel exit $(x=L)$; the radial velocity was specified as $v=0$ at these locations. Finally, no-slip boundary conditions were applied at the channel wall for both velocity components. 


\subsection{Chemical kinetics}

A one-step catalytic reaction was adapted for the present catalysts, from the original expression provided by the catalyst supplier (having been derived using isothermal tests in a fixed-bed, catalytic reactor filled with catalyst powder). The reaction rate was of the general form:

$$
\begin{aligned}
R_{\mathrm{C}} & (\mathrm{mol} / \mathrm{g} \mathrm{Cat} / \mathrm{s}) \\
= & \frac{k_{1}\left[\mathrm{CH}_{4}\right]^{0.40}\left[\mathrm{O}_{2}\right]^{0.25}}{1+k_{2}\left[\mathrm{CO}_{2}\right]^{0.1}+k_{3}\left[\mathrm{H}_{2} \mathrm{O}\right]^{0.25}}
\end{aligned}
$$

where $k_{i}=A_{i} \exp \left(-E_{i} / R T_{\mathrm{W}}\right)$. The denominator of Eq. (11) accounted for the observed inhibition effects of water and carbon dioxide on the heterogeneous reactions. The specific values of the reaction parameters of particular interest in the present investigation were $A_{1}=846 \mathrm{~mol}^{0.35} \mathrm{~m}^{1.95} / \mathrm{g} \mathrm{Cat} / \mathrm{s}$ and $E_{1}=77.7 \mathrm{~kJ} / \mathrm{mol}$. The known catalyst loading (g Cat $/ \mathrm{m}^{2}$ ) was used to convert the rate $R_{\mathrm{C}}$ to standard surface reaction units of $\mathrm{mol} / \mathrm{m}^{2} / \mathrm{s}$ (and hence the pre-exponential $A_{1}$ to units of $\mathrm{mol}^{0.35} / \mathrm{m}^{0.05} / \mathrm{s}$ ). The value of $A_{1}$ was adjusted using experimental data acquired at various pressures, inlet temperatures and catalyst lengths, in an effort to obtain a more global kinetic expression. Adjustment was also necessary for taking into account the differences between the bench-scale reactor of the catalyst supplier (upon which the original expression is based), and the lab-scale reactor used in the present investigation.

In order to ascertain the influence of gaseous chemistry, the $C_{1}$ scheme of Warnatz and Maas [10] was used to determine whether gaseous combustion was important under the test conditions of Table 1. Predictions of homogeneous ignition using this scheme have shown very good agreement (within 10\%) with homogeneous ignition distances measured within catalytic channels, at pressures up to 6 bar [11]; somewhat larger deviations $(>25 \%)$ were observed at pressures between 8 and 10 bar. Computations were carried out for both catalytically active and inert channels. The latter were more susceptible to homogeneous ignition due to the associated near-wall fuel excess. It is noted that in the simulations of the catalytically active channels, the absence of radical heterogeneous reactions provided a conservative estimate of homogeneous ignition since the radical adsorption/desorption re- actions inhibited gas-phase combustion [7,12]. No homogeneous ignition occurred in both active and inert channel simulations, and, moreover, the overall contribution of the homogeneous pathway to fuel consumption was minimal. Absence of gas-phase combustion was also supported by examination of the catalyst structures after the experiments; no signs indicative of overheating, were observed.

\subsection{Solution algorithm}

A finite-volume approach was employed; the discretized, algebraic, gas-phase transport equations (Eqs. (1)-(5)) were solved iteratively using an ADI algorithm [13], whilst the SIMPLER method [14] resolved the velocity-pressure coupling. Gravity was not important for the Reynolds numbers of this study and was, therefore, not included. An orthogonal, staggered grid of up to $160 \times 24$ points (in $x$ and $r$, respectively) was used, with finer grid spacing near the wall $(r=$ $\left.R_{\mathrm{h}}\right)$ and the channel entrance $(x=0)$. Surface and gas-phase chemistries were coupled via the interfacial boundary conditions of Eqs. (8) and (9), which were solved after each gas-phase iteration was completed. The CHEMKIN database was used to evaluate thermodynamic [15] and transport [9] properties. Surface and gaseous reaction rates were evaluated with CHEMKIN [16] and Surface CHEMKIN [17], respectively.

\section{Results and discussion}

Modeling work was performed in two distinct stages, and upon two different catalysts. The purpose of the first study, involving catalyst A, was to derive a method of determining feasible values for the reaction parameter $A_{1}$. Although this catalyst was not ideal, the numerical work highlighted parameters that influence the overall catalytic conversion. Catalyst B was subsequently designed in light of these findings, and the numerical methodology of part A was applied and verified using the experimental results from the more stable catalyst $\mathrm{B}$.

\subsection{Catalyst a tests and simulations}

The methodology for the determination of the reaction parameter $A_{1}$ is firstly illustrated using test cases 


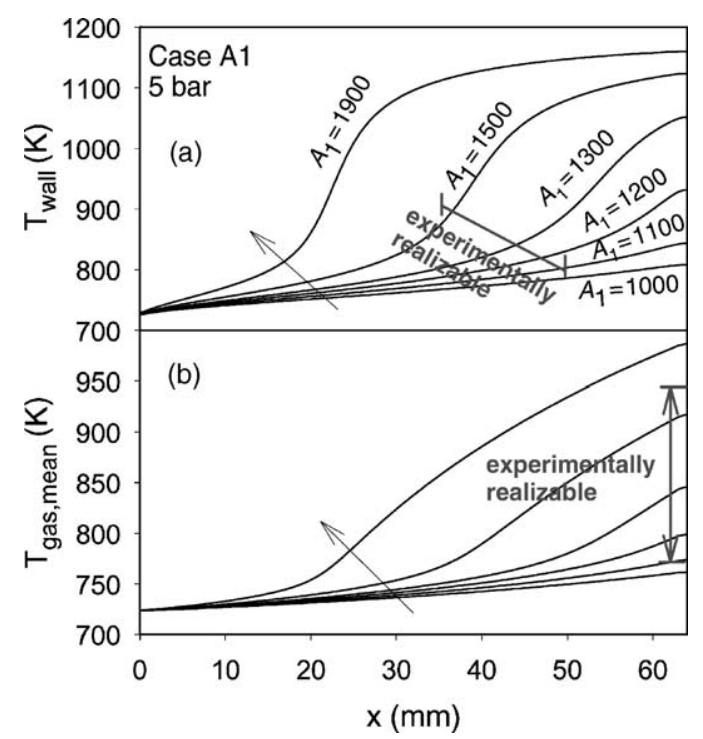

Fig. 3. Streamwise profiles of the computed wall temperatures $T_{\mathrm{W}}$ (a) and of the mean gas temperatures (b) for case A1 (see Table 1) and for various values of the catalytic kinetic parameter $A_{1}$ (see the kinetic rate expression of Eq. (11)). The arrows point to the direction of increasing $A_{1}$, which are the same in both (a) and (b). The vertical arrow in (b) indicates the range of the measured exit temperatures.

A1 and A2 (see Table 1) pertaining to catalyst A. Fig. 3 provides the predicted streamwise, mean gas and wall temperature profiles of case A1, for various values of the pre-exponential parameter $A_{1}$. The corresponding profiles of case A2 are illustrated in Fig. 4. Figs. 3b and $4 \mathrm{~b}$ further indicate the spread of the experimental, catalyst gas exit temperatures, as measured for cases A1 and A2 during a number of cycles. The scatter in measured exit temperatures reflects the slow deactivation of catalyst A over repeated cycles. Fig. 5 provides a comparison of the first and last test cycles performed at condition $\mathrm{A} 1$; short-term instability, as well as long-term deactivation, is witnessed. Clearly, this type of behavior is unsuitable for use in gas turbines. An ideal catalyst would be characterized by the conversion versus time plot shown in Fig. 5b, but after the first run (rather than the fifth). As seen in Figs. $3 \mathrm{~b}$ and $4 \mathrm{~b}$, the measured temperature rise at the exit, $\Delta T\left(=T_{\mathrm{L}, \mathrm{m}}-T_{\mathrm{in}}\right)$, ranged from 225 to $45 \mathrm{~K}$ in case $\mathrm{A} 1$ and from 142 to $33 \mathrm{~K}$ in case $\mathrm{A} 2$. The spectrum of acceptable $A_{1}$ values for both cases was $1100-1300 \mathrm{~mol}^{0.35} / \mathrm{m}^{0.05} / \mathrm{s}$, a reasonably narrow span

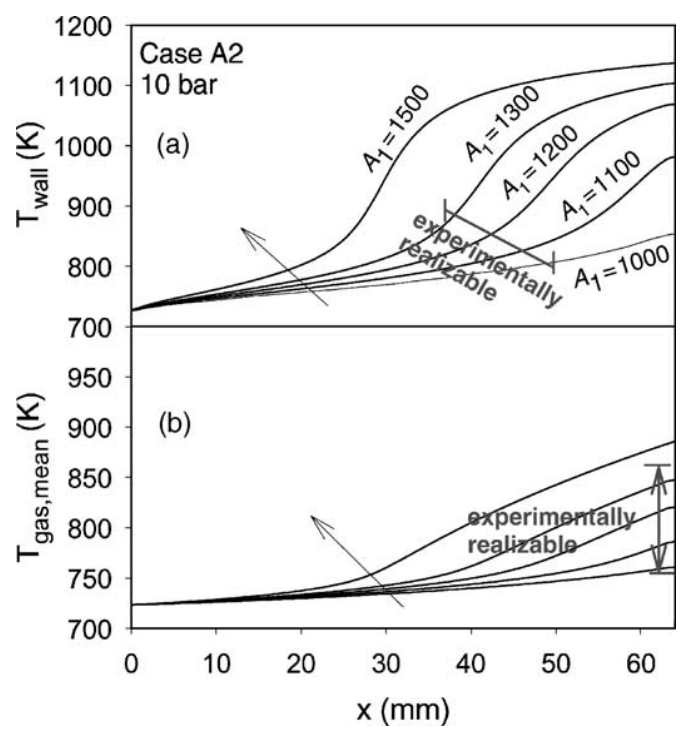

Fig. 4. Streamwise profiles of the computed wall temperatures $T_{\mathrm{W}}$ (a) and of the mean gas temperatures (b) for case A2 (see Table 1) and for various values of the catalytic kinetic parameter $A_{1}$ (see the kinetic rate expression of Eq. (11)). The arrows point to the direction of increasing $A_{1}$, which are the same in both (a) and (b). The vertical arrow in (b) indicates the range of the measured exit temperatures.

for a kinetic parameter; $A_{1}=1200 \mathrm{~mol}^{0.35} / \mathrm{m}^{0.05} / \mathrm{s}$ was chosen as a representative value for catalyst $\mathrm{A}$. Such a value for $A_{1}$ yielded predicted $\Delta T=75$ and $\Delta T=97 \mathrm{~K}$ for cases $\mathrm{A} 1$ and $\mathrm{A} 2$, respectively. The temperature rises measured in the last cycle for each case were 100 and $32 \mathrm{~K}$, respectively. This discrepancy between predicted and experimental values is not alarming, given the rather unsteady behavior of the catalyst. No one-step kinetic expression can accurately account for the effects of short-term instabilities and long-term catalytic deactivation. Nevertheless, the principal aim of this analysis lies in the method of determination of a parameter that yields a realistic range of conversions which, when applied to a stable catalyst, is able to reproduce experimental observations.

In order to gauge the extent of the role of kinetics in the catalyst, the mass-transport-limited (i.e. maximum attainable) conversions were also computed for cases A1 and A2. The diffusion-controlled results were obtained by setting the parameter $A_{1}$ to an artificially high value and then verifying that the solution did not change with a further increase of $A_{1}$. 

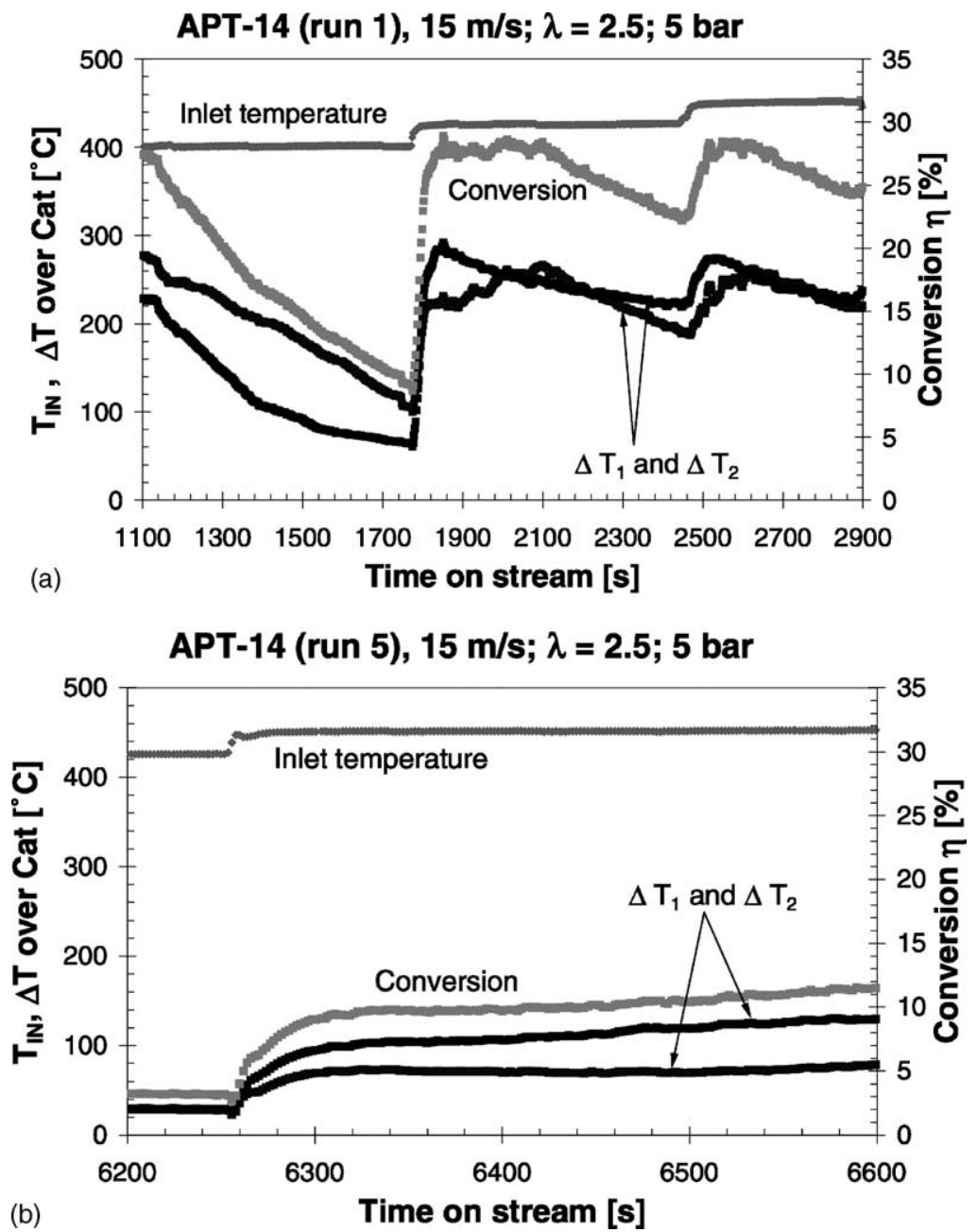

Fig. 5. Comparison of (a) first and (b) last experimental test cycles at condition A1. $\Delta T_{1}$ and $\Delta T_{2}$ are the temperature rises across the catalyst, defined by $\Delta T_{1}=T_{\text {out, } 1}-T_{\text {in }}$ and $\Delta T_{2}=T_{\text {out, } 2}-T_{\text {in }}$, respectively (see Fig. 1b). The absolute conversion $\eta$ is computed using the average catalytic temperature rise $\Delta T=(1 / 2)\left(\Delta T_{1}+\Delta T_{2}\right)$.

Typically, a value of $A_{1}=5 \times 10^{5} \mathrm{~mol}^{0.35} / \mathrm{m}^{0.05} / \mathrm{s}$ was sufficient to obtain a kinetic-free solution. The mass-transport-limited temperature rises were $\Delta T_{\max , A_{1}}=352$ and $\Delta T_{\max , A_{2}}=263 \mathrm{~K}$ (corresponding to maximum fuel conversions of $\sim 78$ and $\sim 58 \%$, respectively, in each coated channel). A comparison of the measured values with the maximum conversions clearly indicates that the surface reactions were far from being diffusion-limited.

Several observations can be made regarding the measured and predicted $\Delta T$ of Figs. 3 and 4 (based on $\left.A_{1}=1200 \mathrm{~mol}^{0.35} / \mathrm{m}^{0.05} / \mathrm{s}\right)$. Firstly, the wall temper- ature profiles of Figs. 3a and 4a indicated that nearly half of the channel $(\sim 35 \mathrm{~mm})$ contributed minimally to the total temperature rise, suggesting a weak surface reactivity in that part of the channel. For the given inlet conditions of cases $\mathrm{A} 1$ and $\mathrm{A} 2$ and the specific channel geometry, the catalytic reactor operated at the verge of light-off, as nearly $80 \%$ of the temperature rise was attained in the rear $14 \mathrm{~mm}$. The observed unstable performance of catalyst A (see Fig. 5) was thus not solely due to intrinsic catalyst deactivation, but also to the aforementioned, highly undesirable, operating mode. The computations further revealed 
a large sensitivity of the temperature rise to small variations in operating parameters ( $U_{\text {in }}$ and especially $T_{\text {in }}$ ); for example, a $10 \mathrm{~K}$ rise in the inlet temperature resulted in a $65 \mathrm{~K}$ increase in $\Delta T$ (for $A_{1}=$ $1200 \mathrm{~mol}^{0.35} / \mathrm{m}^{0.05} / \mathrm{s}$ ). The predictions of Figs. 3 and 4 have also shown that an increase in pressure (and hence in mass throughput) causes gains in both absolute $(\Delta T)$ and fractional conversions $\left(\Delta T / \Delta T_{\max }\right)$. For example, using $A_{1}=1200 \mathrm{~mol}^{0.35} / \mathrm{m}^{0.05} / \mathrm{s}, \Delta T_{A_{1}}=$ $75 \mathrm{~K}$ and $100 \times \Delta T_{A_{1}} / \Delta T_{\max , A_{1}}=21.3 \%$, and $\Delta T_{A_{2}}=97 \mathrm{~K}$ and $100 \times \Delta T_{A_{2}} / \Delta T_{\max , A_{2}}=36.8 \%$. This performance appeared to be counter-intuitive since the fuel conversion in laminar channel flows is (at least under mass-transport-limited operation) inversely proportional to the incoming Reynolds number (see Ref. [12]). However, the observed gain in fuel conversion with increasing pressure can be readily explained from kinetic and transport considerations. The kinetic expression in Eq. (11) reveals that surface reactions accelerate with pressure. Heat and mass-transfer rates in the present channels are highly non-uniform due to the strong radial and axial gradients of all vector and scalar quantities. Despite the linear relationship between mass flow rate and pressure, the rate of surface heat generation appears to increase faster than the local heat transfer to the fluid, thereby causing the fuel conversion to increase, and the light-off distance to diminish, with pressure. This is further illustrated in Fig. 6, where radial profiles of the methane mass fraction and temperature are presented at three selected axial distances $(x=10,30$ and $64 \mathrm{~mm}$ ), for both cases A1 and A2. The near-wall methane levels in case A2 are always lower than those of case A1, indicating greater surface reactivity in the higher-pressure case A2 (see Fig. 6a). Conversely, the near-wall temperature is larger in the higher-pressure case A2 (see Fig. 6b).

The bulk of the heterogeneous fuel conversion in cases A1 and A2 was shown to occur near the exit of the catalytic reactor, due to operation on the verge of light-off. Longer channels appeared to be necessary to improve conversion. Computations were therefore performed with an increased channel length of $L=90 \mathrm{~mm}$; the resulting mean gas and wall temperatures are depicted in Fig. 7 (using $A_{1}=$ $1200 \mathrm{~mol}^{0.35} / \mathrm{m}^{0.05} / \mathrm{s}$ ). The attained temperature gains were considerably higher than the $L=64 \mathrm{~mm}$ results of Figs. 3 and $4 ; \Delta T_{A_{1}}^{\prime}=195$ and $\Delta T_{A_{2}}^{\prime}=172 \mathrm{~K}$.

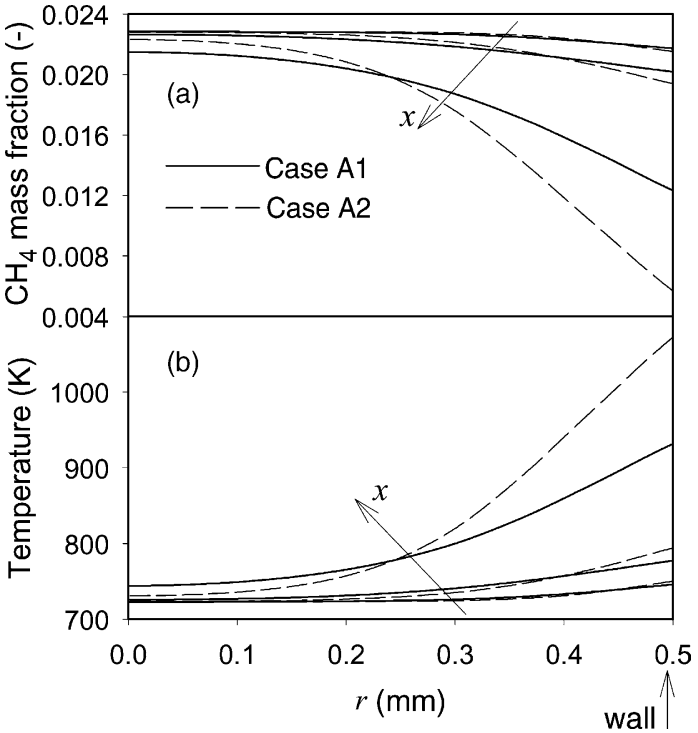

Fig. 6. Radial profiles of the computed methane mass fractions (a) and of the gas temperatures (b) for cases A1 (solid lines) and A2 (dashed lines) of Table 1, for three streamwise distances $x=10$, 30 and $64 \mathrm{~mm}$. The arrows point to the direction of increasing axial distance. All computations refer to a value of the kinetic parameter $A_{1}=1200 \mathrm{~mol}^{0.35} / \mathrm{m}^{0.05} / \mathrm{s}$ (see Eq. (11)).

The associated mass-transport-limited temperature rises were $\Delta T_{\max , A_{1}}^{\prime}=396$ and $\Delta T_{A_{2}}^{\prime}=309 \mathrm{~K}$ (corresponding to maximum fuel conversions of 87 and $68 \%$, respectively, in each active channel), resulting

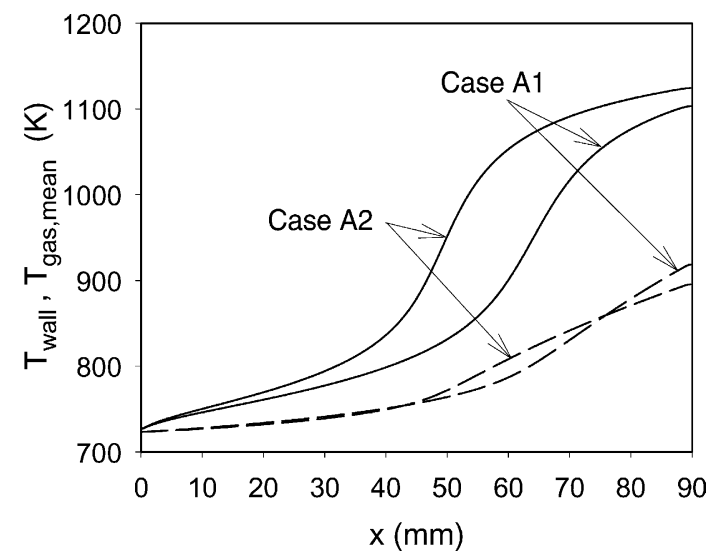

Fig. 7. Streamwise profiles of the computed wall temperatures (solid lines) and mean gas temperatures (dashed lines) for the conditions of cases A1 and A2 (Table 1) but with an increased channel length of $90 \mathrm{~mm}$. All computations refer to a value of the kinetic parameter $A_{1}=1200 \mathrm{~mol}^{0.35} / \mathrm{m}^{0.05} / \mathrm{s}$ (see Eq. (11)). 
in fractional conversions of $100 \times \Delta T_{A_{1}} / \Delta T_{\max , A_{1}}=$ $49 \%$ and $100 \times \Delta T_{A_{2}} / \Delta T_{\max , A_{2}}=55 \%$. The improved performance was achieved without catalyst overheat or initiation of gaseous combustion; the surface temperatures of Fig. 7 did not exceed $1125 \mathrm{~K}$ (note that the decomposition temperatures of $\mathrm{PdO}$ are $\sim 1160 \mathrm{~K}$ at 5 bar and $\sim 1210 \mathrm{~K}$ at $10 \mathrm{bar}$ ). The penalty of the increased length was a small increase in pressure drop, for example, $100 \times \Delta p / p$ increased from 0.17 to $0.24 \%$ in case A2.

The analysis of this section has provided the methodology for determining catalytic kinetic parameters from limited experimental data, such that numerical tools can be used to model a number of geometric configurations using that particular catalyst. Furthermore, shorter light-off distances were promoted at higher pressures, due to enhanced catalytic activity. Long sections of the channels were seen to convert miniscule amounts of fuel, thereby indicating that measures are required for boosting the effectiveness of the catalytically active surface area. The crucial effect of channel length on fuel conversion was identified. Although the performance of a longer catalyst, as depicted in Fig. 7, could be desirable in real-life applications, catalyst A was insufficiently stable for practical use in a gas turbine combustor. Nevertheless, the knowledge obtained from the tests and simulations of catalyst A, was subsequently applied to the testing and modeling of catalyst B, which was inherently stable and had real potential for practical, GT applications.

\subsection{Catalyst B tests and simulations}

Catalyst B was selected for its excellent thermal stability over extended time periods; there was little vari- ation of measured gas temperature with cycle number. The temperature and conversion versus time behavior was similar to that shown in Fig. 5b. Relative to catalyst $\mathrm{A}$, the catalytic loading was somewhat reduced and the formulation slightly different; nevertheless, it obeyed the kinetic rate expression of Eq. (11). In order to compensate for the lower activity, the hydraulic diameter of the channels (both active and inert) was increased to $1.2 \mathrm{~mm}$. Light-off was aided in this instance because the radial transport rates in laminar channel flow scale with $D_{\mathrm{h}}^{-2}$ (see discussion in Ref. [12]).

A number of parameters (channel length, pressure and inlet temperature) were varied during the investigation of catalyst B (see Table 1). Both short $(64 \mathrm{~mm})$ and long $(136 \mathrm{~mm})$ channels were used, given the importance of length found in the catalyst A investigation (where $90 \mathrm{~mm}$ was shown to induce large improvements compared to $64 \mathrm{~mm}$ ). The length of the long channels was specified to be greater than $90 \mathrm{~mm}$ to compensate for the less favorable surface-to-volume ratio dictated by the larger hydraulic diameter. The other parameters varied were pressure $(5,10$ and 15 bar) and inlet temperature (698 and $723 \mathrm{~K}$ ). The agreement between experimental and numerical results was excellent for gas exit temperatures smaller than $948 \mathrm{~K}$; these cases are described in Tables 1 and 2 , and are the focus of the present investigation. In the instances where the gas exit temperatures were higher than $948 \mathrm{~K}$ (due to inlet temperatures of $798 \mathrm{~K}$ and above-not shown in Table 2), the agreement was worse. The root of this discrepancy is believed to be the catalyst deactivation due to the transformation of active $\mathrm{PdO}$ to inactive $\mathrm{Pd}$.

Determination of the kinetic parameter $A_{1}$ from tests B1 through B5, involved the same procedure as for catalyst $\mathrm{A}$; this process was aided by the stable

Table 2

Measured and predicted temperature rise in catalyst B tests ${ }^{\mathrm{a}}$

\begin{tabular}{lcclc}
\hline Case & $\Delta T$ measured $(\mathrm{K})$ & $\Delta T$ computed $(\mathrm{K})$ & $\Delta T_{\max }$ computed $(\mathrm{K})$ & $100 \times \Delta T / \Delta T_{\max }$ computed $(\%)$ \\
\hline B1 & 13.7 & 13.5 & 326.5 & 4.1 \\
B2 & 38.4 & 37.5 & 415.8 & 9.0 \\
B3 & 164.8 & 161.4 & 419.9 & 38.4 \\
B4 & 140.0 & 160.1 & 340.7 & 47.0 \\
B5 & 142.1 & 148.6 & 287.6 & 51.6 \\
\hline
\end{tabular}

${ }^{\text {a }}$ All predictions refer to a value of the kinetic parameter $A_{1}=900 \mathrm{~mol}^{0.35} / \mathrm{m}^{0.05} / \mathrm{s}$. The $\Delta T_{\max }$ provides the mass-transport-limited computed temperature rise. 


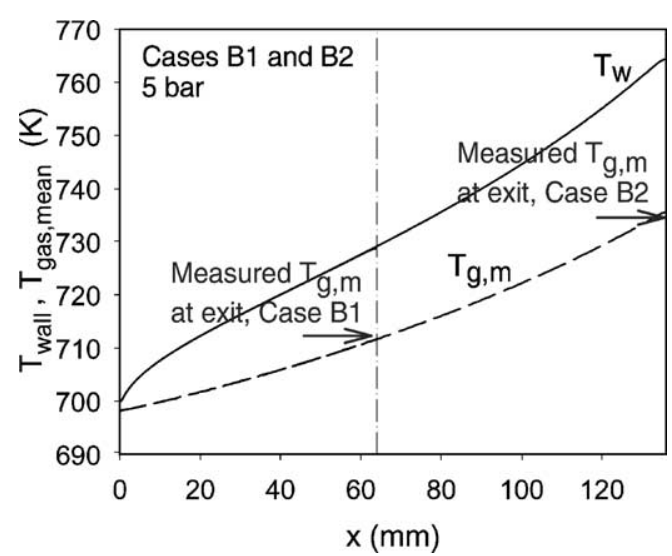

Fig. 8. Streamwise profiles of the computed wall temperatures (solid lines) and mean gas temperatures (dashed lines) for cases B1 (channel length $L=64 \mathrm{~mm}$ ) and B2 (channel length $L=136 \mathrm{~mm}$ ) of Table 1 . The solutions of both cases practically coincide up to $x=64 \mathrm{~mm}$. All computations refer to a value of the kinetic parameter $A_{1}=900 \mathrm{~mol}^{0.35} / \mathrm{m}^{0.05} / \mathrm{s}$ (see Eq. (11)).

behavior (i.e. constant conversion with time) of the catalyst. Comparisons between measurements and predictions revealed that the optimum value of the kinetic parameter was $A_{1}=900 \mathrm{~mol}^{0.35} / \mathrm{m}^{0.05} / \mathrm{s}$. It is worth emphasizing that a single value for this parameter can account for variations in inlet temperature, pressure and channel length. The measured and computed (the latter with $A_{1}=900 \mathrm{~mol}^{0.35} / \mathrm{m}^{0.05} / \mathrm{s}$ ) temperature rise $\Delta T_{B_{i}}$, maximum (mass-transport-limited) temperature rise $\Delta T_{\max , B_{i}}$, and fractional conversion $100 \times T_{B_{i}} / \Delta T_{\max , B_{i}}$ are shown in Table 2 for all cases $(i=1,2, \ldots, 5)$. Fig. 8 illustrates the streamwise profiles of the mean gas and wall temperature for cases B1 and B2 (effect of varying the channel length at 5 bar) and Fig. 9 shows the corresponding profiles for cases B3-B5 (effect of varying the pressure, for long channels). The profiles of cases B1 and B2 in Fig. 8 essentially coincided up to $x=64 \mathrm{~mm}$ and showed excellent agreement with the measured exit gas temperature (see also Table 2). The minimal fuel conversion (only $9.0 \%$ fractional conversion for the longer channel) was due to the low inlet temperature $(698 \mathrm{~K})$. Appreciably larger fractional conversions, ranging from 38.4 to $51.6 \%$, were observed at the higher inlet temperature $\left(T_{\text {in }}=723 \mathrm{~K}\right)$ of cases B3-B5. The agreement between measured and predicted $\Delta T$ was particularly good in cases B3 and B5, but somewhat over-predicted (by $20 \mathrm{~K}$ ) in case $\mathrm{B} 4$.

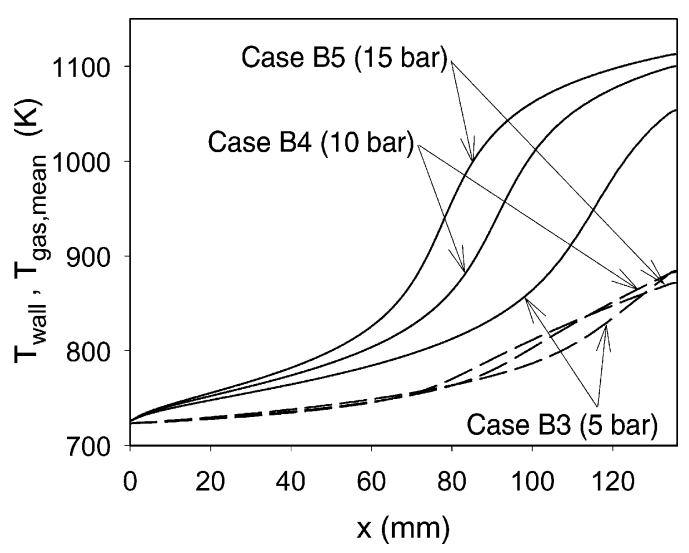

Fig. 9. Streamwise profiles of the computed wall temperatures (solid lines) and mean gas temperatures (dashed lines) for cases B3-B5 of Table 1. All computations refer to a value of the kinetic parameter $A_{1}=900 \mathrm{~mol}^{0.35} / \mathrm{m}^{0.05} / \mathrm{s}$ (see Eq. (11)).

Both experiments and predictions indicated that the achieved temperature rise was virtually independent of pressure. Fig. 9 clearly illustrates the effect of pressure on computed surface and gas temperatures. The higher wall temperatures are attributed to the enhanced surface activity due to increasing pressure (as in the catalyst A cases), but the larger mass throughputs resulted in little change of the gas temperature. The slower surface kinetics at lower pressures is also illustrated in Figs. 10 and 11. Radial profiles of the methane mass fractions are shown in Fig. 10 for three selected longitudinal distances $(x=30,70$ and $136 \mathrm{~mm}$ ); they indicate, at a given axial location, lower $\mathrm{CH}_{4}$ wall concentrations for the higher-pressure cases. The centerline $\mathrm{CH}_{4}$ levels, on the other hand, are greater in the higher-pressure cases, reflecting the larger mass flow rates. The temperature profiles of Fig. 11 reveal that, as pressure becomes larger, centerline and wall temperatures decrease and increase, respectively. The net result was that the mean gas exit temperatures remained relatively unaffected by the pressure.

The discussion pertaining to catalyst $\mathrm{B}$ has shown that it is possible for a single kinetic parameter to be used to accurately predict gas exit temperatures for catalytic reactors operating under a number of different configurations and conditions, including varying lengths, pressures and inlet temperatures. Furthermore, catalyst B, coupled with the reactor geometry of cases B3-B5, demonstrates a number of qualities required for use in practical, high-pressure applica- 


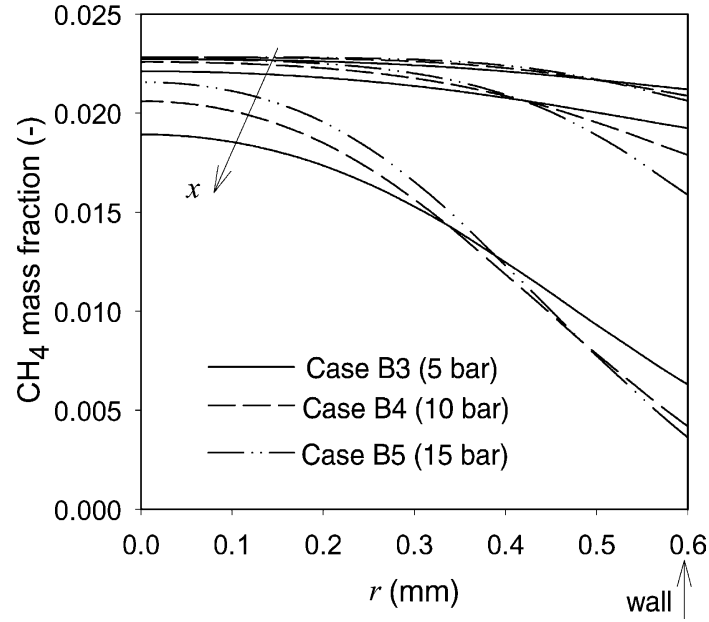

Fig. 10. Radial profiles of the computed methane mass fractions for cases B3 (solid lines), B4 (dashed lines) and B5 (dashed double-dotted lines) of Table 1, for three streamwise distances $x=30,70$ and $136 \mathrm{~mm}$. The arrows point to the direction of increasing axial distance. All computations refer to a value of the kinetic parameter $A_{1}=900 \mathrm{~mol}^{0.35} / \mathrm{m}^{0.05} / \mathrm{s}$ (see Eq. (11)).

tions; the fractional fuel conversion is relatively constant, and the $\Delta T$ is nearly independent of pressure. Additionally, the surface temperatures remained at acceptable levels ( $<1120 \mathrm{~K}$, see Fig. 9), which is below

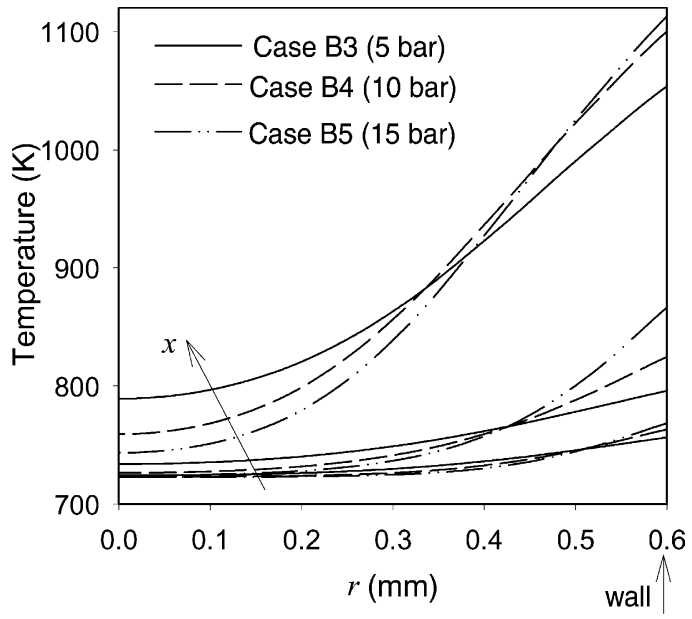

Fig. 11. Radial profiles of the computed gas temperatures for cases B3 (solid lines), B4 (dashed lines) and B5 (dashed double-dotted lines) of Table 1, for three axial distances $x=30,70$ and $136 \mathrm{~mm}$. The arrows point to the direction of increasing streamwise distance. All computations refer to a value of the kinetic parameter $A_{1}=900 \mathrm{~mol}^{0.35} / \mathrm{m}^{0.05} / \mathrm{s}$ (see Eq. (11)). the PdO transformation threshold. Finally, the derived value of the kinetic parameter $A_{1}$ only provides agreement with experimental results for cases in which the exit gas temperatures, and hence surface temperatures, are below a certain level ( $948 \mathrm{~K}$ for the former).

\section{Conclusions}

The catalytic combustion of $\mathrm{CH}_{4} /$ air mixtures over Pd-based catalysts was investigated experimentally and numerically at gas turbine relevant conditions (pressures up to 15 bar and inlet temperatures up to $723 \mathrm{~K}$ ) in a reactor comprising of alternately coated channels. A global catalytic reaction rate was deduced, valid for catalyst temperatures below the PdO decomposition temperature. Experiments and predictions have shown that, even in practical applications, a substantial part of the catalyst contributed minimally to the overall steady-state temperature rise (i.e. the light-off distances were a significant fraction of the channel length). This would suggest that an improved design could reduce light-off distances (e.g. coating all the channels in the inlet section). Nevertheless, the catalyst temperatures remained below the PdO decomposition temperature corresponding to each particular pressure. The catalytic activity increased with rising pressure and, with a proper choice of channel length and hydraulic diameter, it resulted in a nearly constant, pressure-independent, absolute temperature gain across the catalytic reactor. It was thus shown that simplified kinetics-extracted from comprehensive sets of experimental data-coupled with a CFD code, provides a powerful tool for shedding light upon the phenomena occurring within a catalytic reactor, and provides very useful indications for design purposes.

\section{Acknowledgements}

Additional support was provided by the Swiss Federal Office of Education and Technology (BBT) via the contract no. KTI 5344.2 EBS.

\section{References}

[1] O. Deutschmann, R. Schmidt, F. Behrendt, J. Warnatz, Numerical modeling of catalytic ignition, Proc. Combust. Inst. 26 (1996) 1747. 
[2] F. Moallemi, G. Batley, V. Dupont, T.J. Foster, M. Pourkashanian, A. Williams, Chemical modeling and measurements of the catalytic combustion of $\mathrm{CH}_{4} /$ air mixtures on platinum and palladium catalysts, Catal. Today 47 (1999) 235.

[3] H. Zhu, G.S. Jackson, Transient modeling for assessing catalytic combustor performance in small gas turbine applications, ASME Paper 2001-GT-0520, 2001.

[4] J.G. McCarty, Kinetics of PdO combustion catalysis, in: Proceedings of the International Workshop on Catalytic Combustion, Tokyo, Japan, 1994, pp. 108-111.

[5] J. Mantzaras, C. Appel, P. Benz, U. Dogwiler, Numerical modelling of turbulent catalytically stabilized channel flow combustion, Catal. Today 59 (2000) 3.

[6] C. Appel, J. Mantzaras, R. Schaeren, R. Bombach, B. Kaeppeli, A. Inauen, An experimental and numerical investigation of turbulent catalytically stabilized channel flow combustion of hydrogen/air mixtures over platinum, Proc. Combust. Inst. 29, in press.

[7] U. Dogwiler, P. Benz, J. Mantzaras, Two-dimensional modelling for catalytically stabilized combustion of a lean methaneair mixture with elementary homogeneous and heterogeneous chemical reactions, Combust. Flame 116 (1999) 243.

[8] R.K. Shah, A.-L. London, Laminar Flow Forced Convection in Ducts, Academic Press, New York, 1978, p. 257.

[9] R.J. Kee, G. Dixon-Lewis, J. Warnatz, M.E. Coltrin, J.A. Miller, A Fortran computer code package for the evaluation of gas-phase multicomponent transport properties, Sandia Report SAND86-8246, July 1996, Reprint.
[10] J. Warnatz, U. Maas, Technische Verbrennung, Springer, New York, 1993, p. 101.

[11] M. Reinke, J. Mantzaras, R. Schaeren, B. Bombach, W. Kreutner, A. Inauen, Homogeneous ignition in high-pressure combustion of methane/air over platinum: comparison of measurements and detailed numerical predictions, Proc. Combust. Inst. 29, in press.

[12] J. Mantzaras, C. Appel, P. Benz, Catalytic combustion of methane/air mixtures over platinum: homogeneous ignition distances in channel flow configurations, Proc. Combust. Inst. 28 (2000) 1349.

[13] S.K. Srivatsa, Cham computer code 201, CORA2-a computer code for axisymmetrical combustion chambers, CHAM TR 201/1, 1977.

[14] S. Patankar, Numerical Heat Transfer and Fluid Flow, Hemisphere, New York, 1980, p. 131.

[15] R.J. Kee, F.M. Rupley, J.A. Miller, The CHEMKIN thermodynamic data base, Sandia Report SAND87-8215B, July 1996, Reprint.

[16] R.J. Kee, F.M. Rupley, J.A. Miller, CHEMKIN II: a Fortran chemical kinetics package for the analysis of gas-phase chemical kinetics, Sandia Report SAND89-8009B, July 1996, Reprint.

[17] M.E. Coltrin, R.J. Kee, F.M. Rupley, Surface CHEMKIN: a Fortran package for analyzing heterogeneous chemical kinetics at the solid surface-gas phase interface, Sandia Report SAND90-8003C, July 1996, Reprint. 